\title{
FONCTIONS PROPRES DE L'OPÉRATEUR DE LAPLACE-BELTRAMI ASSOCIÉ AU BI-DISQUE
}

\author{
TAOUFIQ TAHANI
}

(Received 7 December 1989)

Communicated by C. Sutherland

\begin{abstract}
Soit $D$ l'opérateur de Laplace-Beltrami sur le bi-disque. On démontre que les fonctions $\zeta \rightarrow$ $P_{\lambda}(\zeta, u)$ provenant du noyau de Poisson associé au bi-disque sont les seules solutions réelles normalisées du système

$$
\left.D F=0, \quad F\left(F^{2}\right)=2 c F^{2} \text { (où } c \in \mathbf{R}\right)
$$
\end{abstract}

qui satisfont une certaine hypothèse.

1991 Mathematics subject classification (Amer. Math. Soc.): 43-02, 43 A 75.

\section{Introduction}

Soit $U$ le disque unité de $\mathbb{C}$, ensemble des $z \in \mathbb{C}$ tels que $|z|^{2}=z \cdot \bar{z}<1$, et soit $\partial U=\{z \in \mathbb{C}:|z|=1\}$ le cercle unité, sa frontière.

Soit $X=U \times U$ le bi-disque, muni du produit des metriques de Poincaré (qui n'est autre que sa metrique de Bergman), et soit $\Gamma=\partial U \times \partial U$ sa frontière maximale de Furstenberg (cf: [6]).

Notons par $G_{0}$ le groupe $S U(1,1)$ des matrices $\left(\frac{\alpha}{\beta} \frac{\beta}{\alpha}\right) \in G L(2, \mathbb{C})$ qui sont de déterminant 1 et par $K_{0}=S(U(1) \times U(1))$ son sous groupe compact formé des matrices $\left(\begin{array}{ll}\alpha & 0 \\ 0 & \bar{\alpha}\end{array}\right)$.

Considérons le groupe produit $G=G_{0} \times G_{0}$ qu'on identifie au sous groupe de $G L(4, \mathbb{C})$, formé des matrices $g=\left(\begin{array}{cc}g_{1} & 0 \\ 0 & g_{2}\end{array}\right)$ où $g_{1}, g_{2} \in G_{0}$, et $K$ son

This research was supported by the Australian Research Council.

(C) 1992 Australian Mathematical Society 0263-6115/92 \$A2.00+0.00 
sous groupe formé des matrices $\rho=\left(\begin{array}{cc}\rho_{1} & 0 \\ 0 & \rho_{2}\end{array}\right)$ où $\rho_{1}, \rho_{2} \in K_{0}$.

Le groupe $G$ est un groupe de Lie réel, semi-simple, de centre fini et de rang 2. Il opère transitivement sur $X$ et $\Gamma$ par la formule:

$$
g \cdot \zeta=\left(\begin{array}{cc}
g_{1} & 0 \\
0 & g_{2}
\end{array}\right) \cdot\left(\zeta_{1}, \zeta_{2}\right)=\left(\frac{\bar{\beta}_{1}+\bar{\alpha}_{1} \zeta_{1}}{\alpha_{1}+\beta_{1} \zeta_{1}}, \frac{\bar{\beta}_{2}+\bar{\alpha}_{2} \zeta_{2}}{\alpha+2+\beta_{2} \zeta_{2}}\right)
$$

où $g=\left(\begin{array}{cc}g_{1} & 0 \\ 0 & g_{2}\end{array}\right) \in G$ et $\zeta=\left(\zeta_{1}, \zeta_{2}\right) \in X \cup \Gamma$.

L'opérateur de Laplace-Beltrami dans $X$, invariant par cette action est donné par:

où

$$
D=\left(1-\left|\zeta_{1}\right|^{2}\right)^{2} \frac{\partial^{2}}{\partial \zeta_{1} \partial \bar{\zeta}_{1}}+\left(1-\left|\zeta_{2}\right|^{2}\right)^{2} \frac{\partial^{2}}{\partial \zeta_{2} \partial \bar{\zeta}_{2}}
$$

$$
\frac{\partial}{\partial \zeta_{p}}=\frac{1}{2}\left[\frac{\partial}{\partial x_{p}}-i \frac{\partial}{\partial y_{p}}\right] \text { et } \frac{\partial}{\partial \bar{\zeta}_{p}}=\frac{1}{2}\left[\frac{\partial}{\partial x_{p}}+i \frac{\partial}{\partial y_{p}}\right]
$$

si $\zeta_{p}=x_{p}+i y_{p} \quad(p=1,2)$.

Le noyau de Poisson dans le cas du bi-disque est le produit $P\left(\zeta_{1}, u_{1}\right) P\left(\zeta_{2}, u_{2}\right)$ des noyaux correspondant au cas du disque:

$$
P\left(\zeta_{p}, u_{p}\right)=\frac{1-\left|\zeta_{p}\right|^{2}}{\left|1-\zeta_{p} \bar{u}_{p}\right|^{2}} \quad \text { pour } p=1,2 \text {. }
$$

Ici, un phénomène intéressant se produit. En effet, si $\lambda=\left(\lambda_{1}, \lambda_{2}\right)$ est un point du cercle $\mathscr{C}(0, \sqrt{2} / 2)$ (i.e. $\left.\lambda_{1}^{2}+\lambda_{2}^{2}=1 / 2\right)$ et si $u=\left(u_{1}, u_{2}\right) \in \Gamma$, la fonction $P_{\lambda}$ définie dans $X$ par:

$$
P_{\lambda}(\zeta, b)=\left(P\left(\zeta_{1}, u_{1}\right)\right)^{1 / 2+\lambda_{1}}\left(P\left(\zeta_{2}, u_{2}\right)\right)^{1 / 2+\lambda_{2}}
$$

est harmonique au sens $D P_{\lambda}=0$.

Il est également connu que, pour tout exposant complexe $\mu$, tout $\lambda \epsilon$ $\mathscr{C}(0, \sqrt{2} / 2)$ et tout $u \in \Gamma$ fixés, la fonction sur $X$

$$
\zeta \rightarrow\left(P_{\lambda}(\zeta, u)\right)^{\mu}
$$

vérifie l'équation

$$
D\left(P_{\lambda}^{\mu}(\cdot, u)\right)=\mu(\mu-1)\left(1+\lambda_{1}+\lambda_{2}\right) P_{\lambda}^{\mu}(\cdot, u)
$$

L'objet de ce papier est de donner une réciproque de cette dernière propriété.

Des problèmes analogues à celui-ci ont fait l'objet d'études dans [1], [8], [5] et [4] sur des espaces symetriques de rang 1 et dans [2] sur les variétés riemannienne de dimension supérieure ou egal à 2 .

Dans ce présent travail, on démontre que les $\zeta \rightarrow P_{\lambda}(\zeta, u)$ sont les seules fonctions $F$ réelles normalisées qui vérifient dans $X$ l'équation (1) 
et dont les dérivées secondes à l'origine vérifient une hypothèse (hypothèse (2d) du théorème) dont j'ignore si elle est nécessaire et qui, même si elle apparait compliquées, se simplifie énormément lorsqu'on passe aux coordonnées horisphériques et que l'on fait un certain changement de fonction (voir l'hypothèse $(7 d)$ du lemma 2 . Ce résultat est le suivant:

ThÉREME. Soit $F$ une fonction réelle de classe $C^{2}$ dans $X$, telle que $F(0)=1$, et vérifiant dans $X$ les deux équations:

(2a) $D F=0$

(2b) $D\left(F^{2}\right)=2 c F^{2}$, où $c \in \mathbb{R}$.

Supposons de plus que, pour $j=1$ et 2 , nous ayons:

$$
\left|\operatorname{Re}\left[\left(\frac{\partial F}{\partial \bar{\zeta}_{j}}(0)\right)^{2} \frac{\partial^{2} F}{\partial \zeta_{j}^{2}}(0)-\left|\frac{\partial F}{\partial \zeta_{j}}(0)\right|^{2} \frac{\partial^{2} F}{\partial \zeta_{j} \partial \bar{\zeta}_{j}}(0)\right]\right|=4\left|\frac{\partial F}{\partial \zeta_{j}}(0)\right|^{3} .
$$

Alors il existe $u=\left(u_{1}, u_{2}\right)$ dans $\Gamma$ et $\lambda=\left(\lambda_{1}, \lambda_{2}\right)$ dans $\mathscr{C}(0, \sqrt{2} / 2)$ avec $\lambda_{1}+\lambda_{2}+1=c$ tels que, pour tout $\zeta \in X$, on ait:

$$
F(\zeta)=P_{\lambda}(\zeta, u)=\left[P\left(\zeta_{1}, u_{1}\right)\right]^{1 / 2+\lambda_{1}}\left[P\left(\zeta_{2}, u_{2}\right)\right]^{1 / 2+\lambda_{2}}
$$

\section{Simplification du problème par action du groupe $K$}

Compte-tenu de l'identité:

$$
D\left(F^{2}\right)=2 F \cdot D F+2\left\{\left(1-\left|\zeta_{1}\right|^{2}\right)^{2}\left|\frac{\partial F}{\partial \zeta_{1}}\right|^{2}+\left(1-\left|\zeta_{2}\right|^{2}\right)^{2}\left|\frac{\partial F}{\partial \zeta_{2}}\right|^{2}\right\},
$$

les hypothèses $(2 a)$ et $(2 b)$ équivalent à:

$$
\begin{gathered}
D F=0, \text { et } \\
\left(1-\left|\zeta_{1}\right|^{2}\right)^{2}\left|\frac{\partial F}{\partial \zeta_{1}}\right|^{2}+\left(1-\left|\zeta_{2}\right|^{2}\right)^{2}\left|\frac{\partial F}{\partial \zeta_{2}}\right|^{2}=c F^{2} .
\end{gathered}
$$

On peut supposer que $c \geq 0$; car si $c<0$, l'hypothèse $\left(2 b^{\prime}\right)$ impliquerait que $F \equiv 0$, mais ceci contredirait le fait que $F(0)=1$.

Comme $F$ vaut 1 à l'origine, on a, d'après $\left(2 b^{\prime}\right)$ :

$$
\left|\frac{\partial F}{\partial \zeta_{1}}(0)\right|^{2}+\left|\frac{\partial F}{\partial \zeta_{2}}(0)\right|^{2}=c .
$$

Par conséquent, il existe $\left(r_{1}, r_{2}\right) \in \mathbb{R}^{2}$ et $\left(e^{-i \theta_{1}}, e^{-i \theta_{2}}\right) \in \Gamma$ tels que: $r_{1}^{2}+r_{2}^{2}=$ $c$ et

$$
\frac{\partial F}{\partial \zeta_{1}}(0)=r_{1} e^{-i \theta_{1}}, \quad \frac{\partial F}{\partial \zeta_{2}}(0)=r_{2} e^{-i \theta_{2}}
$$


Pour $(a, b) \in I=\{(0,0) ;(0,1) ;(1,0) ;(1,1)\}$, considérons dans $K$ la matrice:

$$
\rho_{(a, b)}=\left(\begin{array}{ccc}
e^{-i\left(a \pi+\theta_{1}\right) / 2} & & 0 \\
& e^{i\left(a \pi+\theta_{1}\right) / 2} & e^{-i\left(b \pi+\theta_{2}\right) / 2} \\
0 & e^{i\left(b \pi+\theta_{2}\right) / 2}
\end{array}\right) .
$$

Puisque $D$ est invariant par l'action de $G$ et donc par celle de $K$, la fonction $F_{\rho(a, b)}$ définie par:

$$
F_{\rho(a, b)}\left(\zeta_{1}, \zeta_{2}\right)=F\left(\rho(a, b) \cdot\left(\zeta_{1}, \zeta_{2}\right)\right)=F\left(e^{i\left(a \pi+\theta_{1}\right)} \zeta_{1}, e^{i\left(b \pi+\theta_{2}\right)} \zeta_{2}\right)
$$

satisfait aux équations (2a) et (2b). De plus, nous avons:

$$
\begin{aligned}
& \frac{\partial F_{\rho(a, b)}}{\partial \zeta_{1}}(0)=r_{1} e^{i a \pi}=(1-2 a) r_{1}, \\
& \frac{\partial F_{\rho(a, b)}}{\partial \zeta_{2}}(0)=r_{2} e^{i b \pi}=(1-2 b) r_{2} .
\end{aligned}
$$

L'hypothèse (2d) se transforme, pour la fonction $F_{\rho(a, b)}$, en:

$$
r_{j}^{2}\left|\operatorname{Re}\left[\frac{\partial^{2} F_{\rho(a, b)}}{\partial \zeta_{j}^{2}}(0)-\frac{\partial^{2} F_{\rho(a, b)}}{\partial \zeta_{j} \partial \bar{\zeta}_{j}}(0)\right]\right|=\left|r_{j}\right|^{3} ; \quad \text { pour } j=1,2
$$

\section{Passage aux coordonnées horisphériques}

Pour tout $(\tau, t) \in \mathbb{R}^{2}$ et tout $(\xi, \eta) \in \mathbb{R}^{2}$, considérons dans $G$ les matrices:

$$
\begin{aligned}
& a_{(\tau, t)}=\left(\begin{array}{llll}
\operatorname{ch}(\tau / 2) & \operatorname{sh}(\tau / 2) & & \\
\operatorname{sh}(\tau / 2) & \operatorname{ch}(\tau / 2) & & \\
& & \operatorname{ch}(t / 2) & \operatorname{sh}(t / 2) \\
& & \operatorname{sh}(t / 2) & \operatorname{ch}(t / 2)
\end{array}\right) ; \\
& n_{(\xi, \eta)}=\left(\begin{array}{cccc}
1+i \xi / 2 & -i \xi / 2 & & \\
i \xi / 2 & 1-i \xi / 2 & & \\
& & 1+i \eta / 2 & -i \eta / 2 \\
& & i \eta / 2 & 1-i \eta / 2
\end{array}\right) \text {. }
\end{aligned}
$$

On calcule que:

$$
\left(\zeta_{1}, \zeta_{2}\right)=n(\xi, \eta) a_{(\tau, t)} \cdot 0=\left(\frac{e^{\tau}-1+i \xi}{e^{\tau}+1+i \xi}, \frac{e^{t}-1+i \eta}{e^{t}+1+i \eta}\right) .
$$


Cette formule définit les coordonnées horisphériques $(\tau, t \xi, \eta) \in \mathbb{R}^{4} \mathrm{du}$ point $\zeta=\left(\zeta_{1}, \zeta_{2}\right)=n_{(\xi, \eta)} a_{(\tau, t)} \cdot 0$ variant dans $X$.

Pour $u=\left(u_{1}, u_{2}\right) \stackrel{\in}{\in} \Gamma$ et $\zeta=n_{(\xi, \eta)} a_{(\tau, t)} \cdot 0 \in X$, la fonction $P_{\lambda}$, provenant du noyau de Poisson hyperbolique, est donné en coordonnées horisphériques par la formule:

$$
\begin{aligned}
P_{\lambda}(\zeta, u)= & \left|\left(\frac{1-\bar{u}_{1}}{2}\right) e^{\tau / 2}+\left(1-\frac{1-\bar{u}_{1}}{2}(1-i \xi)\right) e^{-\tau / 2}\right|^{-2\left(1 / 2+\lambda_{1}\right)} \\
& \times\left|\left(\frac{1-\bar{u}_{2}}{2}\right) e^{t / 2}+\left(1-\frac{1-\bar{u}_{2}}{2}(1-i \eta)\right) e^{-t / 2}\right|^{-2\left(1 / 2+\lambda_{2}\right)} .
\end{aligned}
$$

En particulier, pour $u=e=(1,1)$, on a la formule remarquablement simple:

$$
P_{\lambda}(\zeta, e)=e^{\left(1 / 2+\lambda_{1}\right) \tau+\left(1 / 2+\lambda_{2}\right) t}
$$

De plus, l'expression de $D$ en coordonnées horisphériques (cf: [3], pour le disque) est:

$$
D=\frac{\partial^{2}}{\partial \tau^{2}}-\frac{\partial}{\partial \tau}+e^{2 \tau} \frac{\partial^{2}}{\partial \xi^{2}}+\frac{\partial^{2}}{\partial t^{2}}-\frac{\partial}{\partial t}+e^{2 t} \frac{\partial^{2}}{\partial \eta^{2}} .
$$

De l'identité:

$$
D\left(F^{2}\right)=2 F \cdot D F+2\left\{\left(\frac{\partial F}{\partial \tau}\right)^{2}+e^{2 \tau}\left(\frac{\partial F}{\partial \xi}\right)^{2}+\left(\frac{\partial F}{\partial t}\right)^{2}+e^{2 t}\left(\frac{\partial F}{\partial \eta}\right)^{2}\right\},
$$

on déduit que, pour tout $(a, b) \in I$, la fonction $F_{\rho(a, b)}$ satisfait aux relations:

$$
\begin{aligned}
\frac{\partial^{2} F_{\rho(a, b)}}{\partial \tau^{2}} & -\frac{\partial F_{\rho(a, b)}}{\partial \tau}+e^{2 \tau} \frac{\partial^{2} F_{\rho(a, b)}}{\partial \xi^{2}}+\frac{\partial^{2} F_{\rho(a, b)}}{\partial t^{2}}-\frac{\partial F_{\rho(a, b)}}{\partial t} \\
& +e^{2 t} \frac{\partial^{2} F_{\rho(a, b)}}{\partial \eta^{2}}=0
\end{aligned}
$$

$$
\left(\frac{\partial F_{\rho(a, b)}}{\partial \tau}\right)^{2}+e^{2 \tau}\left(\frac{\partial F_{\rho(a, b)}}{\partial \xi}\right)^{2}+\left(\frac{\partial F_{\rho(a, b)}}{\partial t}\right)^{2}+e^{2 t}\left(\frac{\partial F_{\rho(a, b)}}{\partial \eta}\right)^{2}=c F_{\rho(a, b)}^{2} .
$$

En utilisant la formule (3), les relations $\left(2 c^{\prime}\right)$ se transforment en:

$$
\begin{aligned}
& \frac{\partial F_{\rho(a, b)}}{\partial \tau}(0)=(1-2 a) r_{1}, \quad \frac{\partial F_{p(a, b)}}{\partial t}(0)=(1-2 b) r_{2}, \\
& \frac{\partial F_{\rho(a, b)}}{\partial \xi}(0)=\frac{\partial F_{\rho(a, b)}}{\partial \eta}(0)=0 .
\end{aligned}
$$

De plus, en exploitant les équations $\left(2 \mathrm{~d}^{\prime}\right)$, on obtient le 
LEMME 1. Il existe $\left(a_{0}, b_{0}\right) \in I$ tel que la fonction $F_{\rho\left(a_{0}, b_{0}\right)}$ satisfait aux équations:

$$
r_{1} \frac{\partial^{2} F_{\rho\left(a_{0}, b_{0}\right)}}{\partial \xi^{2}}(0)=0, \quad r_{2} \frac{\partial^{2} F_{\rho\left(a_{0}, b_{0}\right)}}{\partial \eta^{2}}(0)=0
$$

Démonstration du Lemma 1. Pour une fonction $F$ de classe $C^{2}$ dans $X$, nous avons:

$$
\begin{aligned}
\frac{\partial^{2} F}{\partial \xi^{2}}(0)= & \frac{\partial F}{\partial \zeta_{1}}(0) \frac{\partial^{2} \zeta_{1}}{\partial \xi^{2}}(0)+\frac{\partial F}{\partial \bar{\zeta}_{1}}(0) \frac{\partial^{2} \bar{\zeta}_{1}}{\partial \xi^{2}}(0)+\frac{\partial^{2} F}{\partial \zeta_{1}^{2}}(0)\left(\frac{\partial \zeta_{1}}{\partial \zeta}(0)\right)^{2} \\
& +2 \frac{\partial^{2}}{\partial \zeta_{1} \partial \bar{\zeta}_{1}}(0) \frac{\partial \zeta_{1}}{\partial \xi}(0) \frac{\partial \bar{\zeta}_{1}}{\partial \xi}(0)+\frac{\partial^{2} F}{\partial \bar{\zeta}_{1}^{2}}(0)\left(\frac{\partial \bar{\zeta}_{1}}{\partial \xi}(0)\right)^{2}
\end{aligned}
$$

En utilisant la formule (3), on peut calculer que:

$$
\frac{\partial^{2} \zeta_{1}}{\partial \xi^{2}}(0)=\frac{\partial^{2} \bar{\zeta}_{1}}{\partial \xi^{2}}(0)=\frac{1}{2}, \quad \frac{\partial \zeta_{1}}{\partial \xi}(0)=\frac{i}{2} \quad \text { et } \quad \frac{\partial \bar{\zeta}_{1}}{\partial \xi}(0)=-\frac{i}{2}
$$

Par conséquent, nous avons:

$$
\frac{\partial^{2} F}{\partial \xi^{2}}(0)=\frac{1}{2}\left(\frac{\partial F}{\partial \zeta_{1}}(0)+\frac{\partial F}{\partial \bar{\zeta}_{1}}(0)\right)-\frac{1}{4} \operatorname{Re}\left[\frac{\partial^{2} F}{\partial \zeta_{1}^{2}}(0)-\frac{\partial^{2} F}{\partial \zeta_{1} \partial \bar{\zeta}_{1}}(0)\right] .
$$

Compte tenu de cette dernière égalité et des formules $\left(2 d^{\prime}\right)$ et $\left(2 c^{\prime}\right)$, nous avons pour tout $(a, b) \in I$ :

$$
r_{1}^{2}\left|(1-2 a) r_{1}-\frac{\partial^{2} F_{\rho(a, b)}}{\partial \xi^{2}}(0)\right|=\left|r_{1}\right|^{3}
$$

De même, nous pouvons obtenir:

$$
r_{2}^{2}\left|(1-2 b) r_{2}-\frac{\partial^{2} F_{\rho(a, b)}}{\partial \eta^{2}}(0)\right|=\left|r_{2}\right|^{3}
$$

Le choix de $a_{0}$ est quelconque lorsque $r_{1}=0$, de même que celui de $b_{0}$ lorsque $r_{2}=0$.

Si $r_{1} \neq 0$, nous avons pour tout $(a, b) \in I$ :

$$
\frac{\partial^{2} F_{\rho(a, b)}}{\partial \xi^{2}}(0)=-2 a r_{1} \text { ou } 2(1-a) r_{1} .
$$

De même, si $r_{2} \neq 0$, nous avons pour tout $(a, b) \in I$ :

$$
\frac{\partial^{2} F_{\rho(a, b)}}{\partial \eta^{2}}(0)=-2 b r_{2} \text { ou } 2(1-b) r_{2} \text {. }
$$


En particulier, pour $(a, b)=(0,0)$, on voit bien que la fonction $F$ initiale vérifie les formules:

$$
\begin{aligned}
& \frac{\partial^{2} F}{\partial \xi}(0)=0 \text { ou } 2 r_{1}, \\
& \frac{\partial^{2} F}{\partial \eta^{2}}(0)=0 \text { ou } 2 r_{2} .
\end{aligned}
$$

Mais, d'après (2c) et (5), nous pouvons calculer que pour touta $(a, b) \in I$ :

$$
\begin{aligned}
& \frac{\partial^{2} F_{\rho(1, b)}}{\partial \xi^{2}}(0)=-2 r_{1}+\frac{\partial^{2} F}{\partial \xi^{2}}(0), \\
& \frac{\partial^{2} F_{\rho(a, 1)}}{\partial \eta^{2}}(0)=-2 r_{2}+\frac{\partial^{2} F}{\partial \eta^{2}}(0) .
\end{aligned}
$$

Dans le cas où $r_{1} \neq 0$, on choisit $a_{0}=0$ lorsque $\left(\partial^{2} F / \partial \xi^{2}\right)(0)=0$ et $a_{0}=1$ lorsque $\left(\partial^{2} F / \partial \xi^{2}\right)(0)=2 r_{1}$.

De même, si $r_{2} \neq 0$, on prend $b_{0}=0$ lorsque $\left(\partial^{2} F / \partial \eta^{2}\right)(0)=0$ et $b_{0}=1$ lorsque $\left(\partial^{2} F / \partial \eta^{2}\right)(0)=2 r_{2}$.

Par ces choix et grâce aux formules (6), on a achevé la démonstration du lemme 1 .

\section{Changement de fonction}

Pour démontrer le théorème, on est ramené à prouver que la fonction $F_{\rho\left(a_{0}, b_{0}\right)}$ n'est autre que $\exp \left(\left(1-2 a_{0}\right) r_{1} \tau+\left(1-2 b_{0}\right) r_{2} t\right)$, et par conséquent que:

$$
F\left(\zeta_{1}, \zeta_{2}\right)=\left[P\left(\zeta_{1}, e^{i\left(\theta_{1}+a_{0} \pi\right)}\right)\right]^{\left(1-2 a_{0}\right) r_{1}}\left[P\left(\zeta_{2}, e^{i\left(\theta_{2}+b_{0} \pi\right)}\right)\right]^{\left(1-2 b_{0}\right) r_{2}},
$$

pour tout $\left(\zeta_{1}, \zeta_{2}\right) \in X$.

Faisant un changement de fonction en posant, dans un voisinage $V$ de 0 où celà $\mathrm{a}$ un sens:

$$
G(\tau, t \xi, \eta)=\log \left[\left(F_{\rho\left(a_{0}, b_{0}\right)}\left(n_{(\xi, \eta)} a_{(\tau, t)} \cdot 0\right)\right]-\left(1-2 a_{0}\right) r_{1} \tau-\left(1-2 b_{0}\right) r_{2} t .\right.
$$

Compte-tenu du fait que $F_{\rho\left(a_{0}, b_{0}\right)}(0)=1$, des équations $(4 a),(4 b),(4 c)$ et (4d), des formules:

$$
\begin{aligned}
& \frac{\partial G}{\partial \tau}=\frac{1}{F_{\rho\left(a_{0}, b_{0}\right)}} \frac{\partial F_{\rho\left(a_{0}, b_{0}\right)}}{\partial \tau}\left(1-2 a_{0}\right) r_{1} ; \quad \frac{\partial G}{\partial \xi}=\frac{1}{F_{\rho\left(a_{0}, b_{0}\right)}} \frac{\partial F_{\rho\left(a_{0}, b_{0}\right)}}{\partial \xi} ; \\
& \frac{\partial^{2} G}{\partial \tau^{2}}=\frac{1}{F_{\rho\left(a_{0}, b_{0}\right)}} \frac{\partial^{2} F_{\rho\left(a_{0}, b_{0}\right)}}{\partial \tau^{2}}-\frac{1}{F_{\rho\left(a_{0}, b_{0}\right)}}\left(\frac{\partial F_{\rho\left(a_{0}, b_{0}\right)}}{\partial \tau}\right)^{2} ; \ldots \text { etc. }
\end{aligned}
$$


et de l'ananlyticité de $F_{\rho\left(a_{0}, b_{0}\right)}$ dans $X$, on est ramené à prouver le

Lemme 2. Soit $V$ un voisinage de 0 dans $\mathbb{R}^{4}$, et soit $G=G(\tau, t, \xi, \eta)$ une fonction réelle de classe $C^{2}$ dans $V$, nulle à l'origine et qui satisfait dans $V$ aux hypothèses:

(7a) $\frac{\partial^{2} G}{\partial \tau^{2}}-\frac{\partial G}{\partial \tau}+e^{2 \tau} \frac{\partial^{2} G}{\partial \xi^{2}}+\frac{\partial^{2} G}{\partial t^{2}}-\frac{\partial G}{\partial t}+e^{2 t} \frac{\partial^{2} G}{\partial \eta^{2}}=\alpha+\beta-\alpha^{2}-\beta^{2}$,

(7b)

$$
\begin{gathered}
\left(\frac{\partial G}{\partial \tau}\right)^{2}+e^{2 \tau}\left(\frac{\partial G}{\partial \xi}\right)^{2}+2 \alpha \frac{\partial G}{\partial \tau}+\left(\frac{\partial G}{\partial t}\right)^{2}+e^{2 t}\left(\frac{\partial G}{\partial \eta}\right)^{2}+2 \beta \frac{\partial G}{\partial t}=0, \\
\frac{\partial G}{\partial \tau}(0)=\frac{\partial G}{\partial t}(0)=\frac{\partial G}{\partial \xi}(0)=\frac{\partial G}{\partial \xi}(0)=\frac{\partial G}{\partial \eta}(0)=0, \\
\alpha \frac{\partial^{2} G}{\partial \xi^{2}}(0)=\beta \frac{\partial^{2} G}{\partial \eta^{2}}(0)=0 .
\end{gathered}
$$

Alors $G$ est identiquement nulle dans $V$.

N.B. Nous avons posé $\alpha=\left(1-2 a_{0}\right) r_{1}$ et $\beta=\left(1-2 b_{0}\right) r_{2}$.

\section{Démonstration du Lemme 2}

Solution de l'équation elliptique (7a) (cf. [7, page 52]), la fonction $G$ est analytique réelle dans $V$; elle est donc, dans un voisinage convenable $W$ de 0 , somme de son développement de Taylor:

$$
G(\tau, t, \xi, \eta)=G_{0}+G_{1}(\tau, t, \xi, \eta)+\cdots+G_{k}(\tau, t, \xi, \eta)+\cdots,
$$

où, pour $k \geq 0, G_{k}$ est un polynôme en les variables $\tau, t, \xi$ et $\eta$, qui est soit identiquement nul, soit homogène de degré $k$.

D'après (7c) et (7d), on sait déjà que $G_{0}$ et $G_{1}$ sont identiquement nuls. Dans un premier temps, nous allons montrer que $G_{2} \equiv 0$; puis, par récurrence sur $k$, nous démontrons que $G_{k} \equiv 0$ pour tout $k \geq 2$.

Dans ce qui suit, on introduit les notations:

$$
A=\alpha \frac{\partial}{\partial \tau}+\beta \frac{\partial}{\partial t}, \quad B=\alpha \frac{\partial^{2}}{\partial \xi^{2}}+\beta \frac{\partial^{2}}{\partial \eta^{2}} \quad \text { et } \quad \Delta=\frac{\partial^{2}}{\partial \tau^{2}}+\frac{\partial^{2}}{\partial t^{2}}+\frac{\partial^{2}}{\partial \xi^{2}}+\frac{\partial^{2}}{\partial \eta^{2}} .
$$

Si $E$ est l'un des trois opérateurs venant d'être cités et $p$ un entier supérieur ou égal 1, on note par $E^{p}$ l'opérateur $E \circ E \circ \circ E$ ( $p$ fois). 
Si $F$ et $G$ sont des fonctions de class $C^{1}$ dans un voisinage $V$ de 0 dans $\mathbb{R}^{4}$, on pose

$\nabla F \cdot \nabla G=\frac{\partial F}{\partial \tau} \frac{\partial G}{\partial \tau}+\frac{\partial F}{\partial t} \frac{\partial G}{\partial t}+\frac{\partial F}{\partial \xi} \frac{\partial G}{\partial \xi}+\frac{\partial F}{\partial \eta} \frac{\partial G}{\partial \eta}$ et $\|\nabla F\|^{2}=\nabla F \cdot \nabla F$.

En prenant les composantes homogènes d'order 0 et 1 dans l'équation (7a), puis les composantes homogènes d'order 1 et 2 dans l'équation (7b), on obtient:

$$
\begin{gathered}
\Delta G_{2}=\alpha+\beta-\alpha^{2}-\beta^{2}, \\
\Delta G_{3}=\frac{\partial G_{2}}{\partial \tau}+\frac{\partial G_{2}}{\partial t}-2 \tau \frac{\partial^{2} G_{2}}{\partial \xi^{2}}-2 t \frac{\partial^{2} G_{2}}{\partial \eta^{2}}, \\
A G_{2}=0, \\
A G_{3}=-\frac{1}{2}\left\|\nabla G_{2}\right\|^{2} .
\end{gathered}
$$

Si on applique l'opérateur $A$ à (9) et $\Delta$ à (11), on obtient:

$$
-\frac{1}{2} \Delta\left[\left\|\nabla G_{2}\right\|^{2}\right]=-2 \alpha \frac{\partial^{2} G_{2}}{\partial \xi^{2}}-2 \beta \frac{\partial^{2} G_{2}}{\partial \eta^{2}}
$$

ce qui, d'après (7d), vaut zéro. Le polynôme $G_{2}$ a donc toutes ses dérivées secondes nulls, il est par conséquent.

Avant de poursuivre la démonstration, remarquons tout d'abord que, compte- tenu de (8) et du fait que $G_{2} \equiv 0$, nous avons:

$$
\alpha+\beta=\alpha^{2}+\beta^{2} \text {. }
$$

Donc, lorsque $\alpha=\beta$, le couple $(\alpha, \beta)$ est soit égale à $(0,0)$, soit égal à $(1,1)$. Mais dans le cas où $(\alpha, \beta)=(0,0)$, il est clair que $G$ est nulle. Plus tard, nous distinguerons alors deux cas: $(\alpha, \beta)=(1,1) ; \alpha \neq \beta$.

Par hypothèse de récurrence, supposons que, pour un entier $k \geq 2$, on ait $G_{0} \equiv G_{1} \equiv G_{2} \equiv \cdots \equiv G_{k} \equiv 0$ et montrons que $G_{k+1} \equiv 0$.

En prenant les composantes homogènes d'ordre $m-1$ (où $m$ est un entier $\leq 2 k), 2 k, 2 k+1$ et $2 k+2$ respectivement dans l'équation ( $7 \mathrm{~b}$ ), nous obtenons

$$
A G_{m}=0
$$

pour tout $m \leq 2 k$ (mais puisque $k \geq 2$ et donc $k+2 \leq 2 k$, nous avons en particulier:

$$
\begin{gathered}
A G_{k+1}=A G_{k+2}=0, \\
A G_{2 k+1}=-\frac{1}{2}\left\|\nabla G_{k+1}\right\|^{2} \\
A G_{2 k+2}=-\nabla G_{k+1} \nabla G_{k+2}-\tau\left(\frac{\partial G_{k+1}}{\partial \xi}\right)^{2}-t\left(\frac{\partial G_{k+1}}{\partial \eta}\right)^{2}
\end{gathered}
$$




$$
\begin{aligned}
A G_{2 k+3}= & -\frac{1}{2}\left\|\nabla G_{k+2}\right\|^{2}-2 \tau \frac{\partial G_{k+1}}{\partial \xi} \frac{\partial G_{k+2}}{\partial \xi}-2 t \frac{\partial G_{k+1}}{\partial \eta} \frac{\partial G_{k+2}}{\partial \eta} \\
& -\tau^{2}\left(\frac{\partial G_{k+1}}{\partial \xi}\right)^{2}-t^{2}\left(\frac{\partial G_{k+1}}{\partial \eta}\right)^{2} .
\end{aligned}
$$

Si on applique l'opérateur $A$ aux deux membres de l'égalité (14), puis l'opérateur $A^{2}$ à ceux de (15), il vient, grâce à (13), que:

$$
\begin{aligned}
& A^{2} G_{2 k+1}=0, \\
& A^{3} G_{2 k+2}=0 .
\end{aligned}
$$

D'autre part, en prenant les composantes homogènes d'ordre $m-2$ dans l'équation ( $7 a)$, on obtient:

$$
\left.\Delta G_{k+1}=0 \quad \text { (lorsque } m=k+1\right),
$$

$\Delta G_{k+2}=-2 \tau \frac{\partial^{2} G_{k+1}}{\partial \xi^{2}}-2 t \frac{\partial^{2} G_{k+1}}{\partial \eta^{2}}+\frac{\partial G_{k+1}}{\partial \tau}+\frac{\partial G_{k+1}}{\partial t} \quad$ (lorsque $\left.m=k+2\right)$,

(18) $\Delta G_{m}=\frac{\partial G_{m-1}}{\partial \tau}+\frac{\partial G_{m-1}}{\partial t}-\sum_{p=1}^{m-k-1}\left[\frac{(2 \tau)^{p}}{p !} \frac{\partial^{2} G_{m-p}}{\partial \xi^{2}}+\frac{(2 t)^{p}}{p !} \frac{\partial^{2} G_{m-p}}{\partial \eta^{2}}\right]$

$$
\text { pour } m \geq k+2 \text {. }
$$

En appliquant l'opérateur $A$ à l'équation (18b) et en tenant compte de (12a), il vient que:

$$
B\left(G_{k+1}\right)=0
$$

Maintentant, si on donne à $m$ les valeurs $2 k$ et $2 k+1$ respectivement dans l'équation (18), on obtient:

$$
\Delta\left(G_{2 k}\right)=\frac{\partial G_{2 k-1}}{\partial \tau}+\frac{\partial G_{2 k-1}}{\partial t}-\sum_{p=1}^{k-1}\left[\frac{(2 \tau)^{p}}{p !} \frac{\partial^{2} G_{2 k-p}}{\partial \xi^{2}}+\frac{(2 t)^{p}}{p !} \frac{\partial^{2} G_{2 k-p}}{\partial \eta^{2}}\right],
$$

$$
\Delta\left(G_{2 k+1}\right)=\frac{\partial G_{2 k}}{\partial \tau}+\frac{\partial G_{2 k}}{\partial t}-\sum_{p=1}^{k}\left[\frac{(2 \tau)^{p}}{p !} \frac{\partial^{2} G_{2 k-p+1}}{\partial \xi^{2}}+\frac{(2 t)^{p}}{p !} \frac{\partial^{2} G_{2 k-p+1}}{\partial \eta^{2}}\right] .
$$

$\mathrm{Si}$, pour $q$ un entier compris entre 1 et $k-1$, nous appliquons les opérateurs $A^{q}$ et $A^{q+1}$ respectivement à ces deux dernières équations; nous 
obtenons:

$$
\begin{aligned}
& \Delta\left[A^{q} G_{2 k}\right]=-2^{q}\left\{\alpha^{q}\left(\sum_{p=q}^{k-1} \frac{(2 \tau)^{p-q}}{(p-q) !} \frac{\partial^{2} G_{2 k-p}}{\partial \xi^{2}}\right)\right. \\
& \left.+\beta^{q}\left(\sum_{p=q}^{k-1} \frac{(2 t)^{p-q}}{(p-q) !} \frac{\partial^{2} G_{2 k-p}}{\partial \eta^{2}}\right)\right\}, \\
& \Delta\left[A^{q+1} G_{2 k+1}\right]=-2^{q+1}\left\{\alpha^{q+1}\left(\sum_{p=q}^{k-1} \frac{(2 \tau)^{p-q}}{(p-q) !} \frac{\partial^{2} G_{2 k-p}}{\partial \xi^{2}}\right)\right. \\
& \left.+\beta^{q+1}\left(\sum_{p=q}^{k-1} \frac{(2 t)^{p-q}}{(p-q) !} \frac{\partial^{2} G_{2 k-p}}{\partial \eta^{2}}\right)\right\} .
\end{aligned}
$$

Or, puisque $q \geq 1$, nous avons d'après (12) et (16):

$$
A^{q} G_{2 k}=A^{q+1} G_{2 k+1}=0 .
$$

Par conséquent, si $\alpha \neq \beta$, les équation (22) et (23) impliquent que:

$$
\alpha \sum_{p=q}^{k-1} \frac{(2 \tau)^{p-q}}{(p-q) !} \frac{\partial^{2} G_{2 k-p}}{\partial \xi^{2}}=\beta \sum_{p=q}^{k-1} \frac{(2 t)^{p-q}}{(p-q) !} \frac{\partial^{2} G_{2 k-p}}{\partial \eta^{2}}=0,
$$

pour tous $1 \leq q \leq k-1$.

En donnant à $q$ les valeurs $k-1$, puis $k-2, \ldots$, puis 1 dans l'équation (24), il vient que:

$$
\begin{gathered}
\alpha \frac{\partial^{2} G_{k+1}}{\partial \xi^{2}}=\cdots=\alpha \frac{\partial^{2} G_{2 k-1}}{\partial \xi^{2}}=0, \\
\beta \frac{\partial^{2} G_{k+1}}{\partial \eta^{2}}=\cdots=\beta \frac{\partial^{2} G_{2 k-1}}{\partial \eta^{2}}=0 .
\end{gathered}
$$

En reportant ceci dans (20) et (21), on obtient les relations:

$$
\begin{gathered}
\Delta G_{2 k}=\frac{\partial G_{2 k-1}}{\partial \tau}+\frac{\partial G_{2 k-1}}{\partial t}, \\
\Delta G_{2 k+1}=\frac{\partial G_{2 k}}{\partial \tau}+\frac{\partial G_{2 k}}{\partial t}-2 \tau \frac{\partial^{2} G_{2 k}}{\partial \xi^{2}}-2 t \frac{\partial^{2} G_{2 k}}{\partial \eta^{2}} .
\end{gathered}
$$

En appliquant l'opèrateur $\Delta \circ A$ à l'équation (26), il vient que:

$$
\Delta^{2}\left(A G_{2 k+1}\right)=\Delta\left[B_{1} G_{2 k}\right]
$$

Or, d'après $(25)$ et $(26)$, nous avons:

$$
\Delta\left[B_{1} G_{2 k}\right]=\frac{\partial}{\partial \tau}\left(B_{1} G_{2 k-1}\right)+\frac{\partial}{\partial t}\left(B_{1} G_{2 k-1}\right)=0 .
$$


Il s'en suit alors que:

$$
\Delta^{2}\left(A G_{2 k+1}\right)=0
$$

Mais, en appliquant l'opérateur $\Delta^{2}$ à l'équation (13), on obtient:

$$
\Delta^{2}\left(A G_{2 k+1}\right)=-\frac{1}{2} \Delta^{2}\left[\left\|\nabla G_{k+1}\right\|^{2}\right] \text {. }
$$

Vu l'équation (18a) et les deux égalités (28) et (29), le polynôme homogène $G_{k+1}$ qu'on avait supposé ou nul ou de degré $k+1 \geq 3$ a toutes ses dérivées troisièmes nulles, par conséquent il est nul.

Désormais, nous supposons que $\alpha=\beta=1$. Dans ce cas l'opérateur $A$ vaut $\partial / \partial \tau+\partial / \partial t$ et $B$ est égal à $\partial^{2} / \partial \xi^{2}+\partial^{2} / \partial \eta^{2}$.

En appliquant l'opérateur $\Delta \circ A$ à l'équation (14) puis l'opérateur $\Delta \circ A^{2}$ à l'équation (15), on obtient:

$$
\Delta\left[A^{2} G_{2 k+2}\right]=-\Delta\left[\left(\frac{\partial G_{k+1}}{\partial \xi}\right)^{2}+\left(\frac{\partial G_{k+1}}{\partial \eta}\right)^{2}\right]
$$

et

$$
\Delta\left[A^{3} G_{2 k+3}\right]=-2 \Delta\left[\left(\frac{\partial G_{k+1}}{\partial \xi}\right)^{2}+\left(\frac{\partial G_{k+1}}{\partial \eta}\right)^{2}\right] \text {. }
$$

Mais

$$
\begin{aligned}
& \Delta\left[\left(\frac{\partial G_{k+1}}{\partial \xi}\right)^{2}+\left(\frac{\partial G_{k+1}}{\partial \eta}\right)^{2}\right] \\
& \quad=\left(\frac{\partial^{2}}{\partial \xi^{2}}+\frac{\partial^{2}}{\partial \eta^{2}}\right)\left(\left\|\nabla G_{k+1}\right\|^{2}\right)-2 \nabla G_{k+1} \cdot \nabla\left(B G_{k+1}\right)=-2 B\left(A G_{2 k+1}\right)
\end{aligned}
$$

d'après (13) et (19).

Par conséquent, nous avons:

$$
\delta\left[a^{3} g_{2 k+3}\right]=2 \delta\left[a^{2} g_{2 k+2}\right]=4 b\left(a g_{2 k+1}\right) .
$$

D'autre part, si on donne à $m$ les valeurs $2 k+2$ puis $2 k+3$ dans l'équation (18), on obtient:

$$
\Delta G_{2 k+2}=A G_{2 k+1}-\sum_{p=1}^{k+1}\left[\frac{(2 \tau)^{p}}{p !} \frac{\partial^{2} G_{2 k+2-p}}{\partial \xi^{2}}+\frac{(2 t)^{p}}{p !} \frac{\partial^{2} G_{2 k+2-p}}{\partial \eta^{2}}\right],
$$

et

$$
\Delta G_{2 k+3}=A G_{2 k+2}=\sum_{p=1}^{k+2}\left[\frac{(2 \tau)^{p}}{p !} \frac{\partial^{2} G_{2 k+3-p}}{\partial \xi^{2}}+\frac{(2 t)^{p}}{p !} \frac{\partial^{2} G_{2 k+3-p}}{\partial \eta^{2}}\right] .
$$

Maintenant, on va appliquer les opérateurs $A^{2}$ et $A^{3}$ respectivement à ces deux dernières équations. 
Nous obtenons pour la première:

$$
\begin{aligned}
\Delta\left(A^{2} G_{2 k+2}\right)= & -A^{2}\left(2 \tau \frac{\partial^{2} G_{2 k+1}}{\partial \xi^{2}}+2 t \frac{\partial^{2} G_{2 k+1}}{\partial \eta^{2}}\right) \\
& -4 \sum_{p=2}^{k+1}\left[\frac{(2 \tau)^{p-2}}{(p-2) !} \frac{\partial^{2} G_{2 k+2-p}}{\partial \xi^{2}}+\frac{(2 t)^{p-2}}{(p-2) !} \frac{\partial^{2} G_{2 k+2-p}}{\partial \eta^{2}}\right] \\
= & -4 B\left(A G_{2 k+1}\right)-4 \sum_{p=1}^{k}\left[\frac{(2 \tau)^{p-1}}{(p-1) !} \frac{\partial^{2} G_{2 k+1-p}}{\partial \xi^{2}}\right. \\
& \left.+\frac{(2 t)^{p-1}}{(p-1) !} \frac{\partial^{2} G_{2 k+1-p}}{\partial \eta^{2}}\right],
\end{aligned}
$$

Mais, si on applique l'opérateur $A$ à l'équation (21), on obtient:

$$
\begin{aligned}
\Delta\left(A G_{2 k+1}\right) & =A\left(-\sum_{p=1}^{k}\left[\frac{(2 \tau)^{p}}{p !} \frac{\partial^{2} G_{2 k+1-p}}{\partial \xi^{2}}+\frac{(2 t)^{p}}{p !} \frac{\partial^{2} G_{2 k+1-p}}{\partial \eta^{2}}\right]\right) \\
& =-2 \sum_{p=1}^{k}\left[\frac{(2 \tau)^{p-1}}{(p-1) !} \frac{\partial^{2} G_{2 k+1-p}}{\partial \xi^{2}}+\frac{(2 t)^{p-1}}{(p-1) !} \frac{\partial^{2} G_{2 k+1-p}}{\partial \eta^{2}}\right] .
\end{aligned}
$$

Il s'ensuit alors que:

$$
\Delta\left(A^{2} G_{2 k+2}\right)=-4 B\left(A G_{2 k+1}\right)+2 \Delta\left(A G_{2 k+1}\right) .
$$

Pour la deuxième, nous avons:

$$
\begin{aligned}
\Delta\left(A^{3} G_{2 k+3}\right)= & -A^{3} a\left(2 \tau \frac{\partial^{2} G_{2 k+2}}{\partial \xi^{2}}+2 t \frac{\partial^{2} G_{2 k+2}}{\partial \eta^{2}}+\frac{(2 \tau)^{2}}{2 !} \frac{\partial^{2} G_{2 k+1}}{\partial \xi^{2}}\right. \\
& \left.+\frac{(2 t)^{2}}{2 !} \frac{\partial^{2} G_{2 k+1}}{\partial \eta^{2}}\right) \\
& -8 \sum_{p=3}^{k+2}\left[\frac{(2 \tau)^{p-3}}{(p-3) !} \frac{\partial^{2} G_{2 k+3-p}}{\partial \xi^{2}}+\frac{(2 t)^{p-3}}{(p-3) !} \frac{\partial^{2} G_{2 k+3-p}}{\partial \eta^{2}}\right] \\
= & -6 B\left(A^{2} G_{2 k+2}\right)-12 B\left(A G_{2 k+1}\right) \\
& -8 \sum_{p=1}^{k}\left[\frac{(2 \tau)^{p-1}}{(p-1) !} \frac{\partial^{2} G_{2 k+1-p}}{\partial \xi^{2}}+\frac{(2 t)^{p-1}}{(p-1) !} \frac{\partial^{2} G_{2 k+1-p}}{\partial \eta^{2}}\right], \\
= & -6 B\left(A^{2} G_{2 k+2}\right)-12 B\left(A G_{2 k+1}\right)+4 \Delta\left(A G_{2 k+1}\right)
\end{aligned}
$$

ce qui, compte-tenu de l'équation (31), devient:

$$
\Delta\left(A^{3} G_{2 k+3}\right)=-6 B\left(A^{2} G_{2 k+2}\right)+2 \Delta\left(A^{2} G_{2 k+2}\right)-4 B\left(A G_{2 k+1}\right) .
$$


Les équations (31) et (32), jointes à l'équation (30), nous donnent:

$$
\begin{gathered}
2 \Delta\left(A G_{2 k+1}\right)=6 B\left(A G_{2 k+1}\right), \\
2 B\left(A G a_{2 k+1}\right)=-3 B\left(A^{2} G_{2 k+2}\right) .
\end{gathered}
$$

En appliquant l'opérateur $B$ à (33) et l'opérateur $\Delta$ à (34) puis en faisant de nouveau appel à l'équation (30), on obtient:

$$
2 \Delta\left[B\left(A G_{2 k+1}\right)\right]=6 B^{2}\left[A G_{2 k+1}\right]=-6 B^{2}\left(A G_{2 k+1}\right) .
$$

Ceci implique que $\Delta\left[B\left(A G_{2 k+1}\right)\right]=0$, c'est à dire, d'après (13), que $\Delta\left[B\left(\left\|\nabla G_{k+1}\right\|^{2}\right)\right]=0$.

Mais, compte-tenu de (18a) et (19), on a:

$$
\Delta\left[B\left(\left\|\nabla G_{k+1}\right\|^{2}\right)\right]=4 \sum_{i, j=1}^{4}\left[\left(\frac{\partial^{3} G_{k_{1}}}{\partial x_{i} \partial x_{j} \partial \xi}\right)^{2}+\left(\frac{\partial^{3} G_{k+1}}{\partial x_{i} \partial x_{j} \partial \eta}\right)^{2}\right],
$$

où l'on a posé $\left(x_{1}, x_{2}, x_{3}, x_{4}\right)=(\tau, t, \xi, \eta)$.

Donc $\partial G_{k+1} / \partial \xi$ et $\partial G_{k+1} / \partial \eta$ sont des polynômes homogènes, nuls ou de degré $k \geq 2$, dont toutes les dérivées secondes par rapport aux variables $(\tau, t, \xi, \eta)$ sont nulles, par conséquent ils sont identiquement nuls. Autrement dit, $G_{k+1}$ est un polynôme en les seules variables $\tau$ et $t$, nul ou de degré $k+1 \geq 3$, or nous avons

$$
\Delta G_{k+1}-B G_{k+1}=\frac{\partial^{2} G_{k+1}}{\partial \tau^{2}}+\frac{\partial^{2} G_{k+1}}{\partial t^{2}}=0 \quad \text { (d'après (18a) et (19)), }
$$

et

$$
\frac{\partial^{2} G_{k+1}}{\partial \tau^{2}}=\frac{\partial^{2} G_{k+1}}{\partial t^{2}}=-\frac{\partial^{2} G_{k+1}}{\partial \tau \partial t} \quad \text { du fait que } A G_{k+1} \text { soit nul. }
$$

Il s'ensuit alors que $G_{k+1} \equiv 0$, ce qui achève la démonstration du Lemme 2 et donc $d u$ théorème.

\section{Acknowledgements}

This work was done while I was in the University of New South Wales (Sydney, Australia). I am thankful for the warm hospitality of the School of Mathematics (U.N.S.W.). I also thank Dr. A. H. Dooley with whom I had fruitful discussions. 


\section{References}

[1] M. Chipot, P. Eymard and T. Tahani, 'Sur les fonctions propres de l'opérateur de Laplace-Beltrami dont le carré est fonction propre', Symposia Mathematica 29 (1988), 111-129.

[2] M. Chipot and V. Oliker, 'Sur une propriété des fonctions propres de l'opérateur de Laplace-Beltrami', preprint.

[3] P. Eymard, 'Le Noyau de Poisson et l'Analyse Harmonique non Euclidienne', Topics in modern harmonic analysis, (Ist. Nazionale di Alta Matematica, Roma, 1983).

[4] T. Kawazoe, 'A property of the eigenfunctions of the Laplace-Beltrami operator on the classical real rank one symmetric spaces', preprint.

[5] T. Kawazoe and T. Tahani, 'A characterization of the Poisson kernel associated with $S U(1, n)$ ', Tokyo J. Math. 11, No. 1 (1988), 37-55.

[6] A. Korányi, A survey of harmonic functions on symmetric spaces, (Proceedings of Symposia in Pure Mathematics, Volume XXXV, Part 1, 1979).

[7] W. Rudin, Function theory in the unit ball of $\mathbb{C}^{n}$, (Springer-Verlag, 1980).

[8] T. Tahani, Fonctions propres de l'operateur de Laplace-Beltrami dans les boules hyporboliques réelles ou complexes, thèse de 3ème cycle, Université de Nancy I (1985).

Université des Sciences et Techniques de

Lille Flandres-Artois

U.F.R. de Mathématiques Pures et Appliquées

59650 Villeneuve d'Ascq

France 